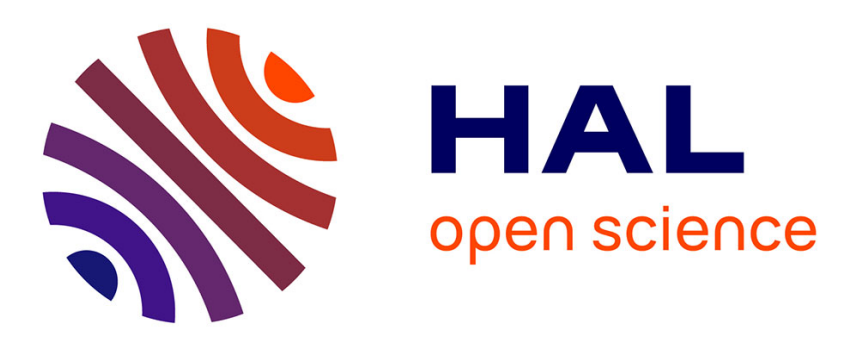

\title{
Quasigeostrophic model of the instabilities of the Stewartson layer in flat and depth-varying containers
}

Nathanaël Schaeffer, Philippe Cardin

\section{To cite this version:}

Nathanaël Schaeffer, Philippe Cardin. Quasigeostrophic model of the instabilities of the Stewartson layer in flat and depth-varying containers. Physics of Fluids, 2005, 17, pp.104111. 10.1063/1.2073547 . hal-00016419

\section{HAL Id: hal-00016419 https://hal.science/hal-00016419}

Submitted on 2 Jan 2013

HAL is a multi-disciplinary open access archive for the deposit and dissemination of scientific research documents, whether they are published or not. The documents may come from teaching and research institutions in France or abroad, or from public or private research centers.
L'archive ouverte pluridisciplinaire HAL, est destinée au dépôt et à la diffusion de documents scientifiques de niveau recherche, publiés ou non, émanant des établissements d'enseignement et de recherche français ou étrangers, des laboratoires publics ou privés. 


\title{
Quasigeostrophic model of the instabilities of the Stewartson layer
}

\author{
By NATHANA E L SCHAEFFER \\ AND PHILIPPE CARDIN † \\ LGIT, Université Joseph Fourier and CNRS, Grenoble, France
}

(Received 18 March 2005; accepted 18 July 2005; published online 31 October 2005)

Detached shear layers in a rotating container, known as Stewartson layers, become instable for a critical shear measured by a Rossby number Ro. To study the asymptotic regime at low Ekman number $E$, a quasigeostrophic (QG) model is developed, whose main original feature is to handle the mass conservation correctly, resulting in a divergent two-dimensional flow, and valid for any container provided that the top and bottom have finite slopes. Asymptotic scalings of the instability are deduced from the linear QG model, extending the previous analysis to large slopes (as in a sphere). For a flat container, the critical Rossby number evolves as $E^{3 / 4}$ and the instability may be understood as a shear instability. For a sloping container, the instability is a Rossby wave with a critical Rossby number proportional to $\beta E^{1 / 2}$, where $\beta$ is related to the slope. A numerical code of the QG model is used to determine the critical parameters for different Ekman numbers and to describe the instability at the onset for different geometries. We also investigate the asymmetry between positive and negative Ro. For flat cylindrical containers, the QG numerical results are directly compared to existing experimental data obtained by Niino \& Misawa (1984) and Früh \& Read (1999). We produced new experimental results of destabilisation of a Stewartson layer in a rotating spheroid, caused by the differential rotation of two disks or a central inner core which are compared to the numerical QG results obtained in a split-sphere, validating the QG model and showing its limits. For spherical shells, the experimental critical curves are compared to the ones obtained by 3D calculation of Hollerbach (2003).

\section{Introduction}

Detached shear layers in rapidly rotating systems (among which we find the so-called Stewartson layers) are of general geophysical interest. They are observed in planetary atmospheres (such as jovian jets) or in oceans (such as the gulf stream). They should also be present in the dynamics of planetary cores and may participate to the dynamo process. To model these geophysical flows, this work considers the geostrophic shear produced by differential rotation of the boundaries of a fast rotating container, in axisymmetric geometries.

Stewartson (1957) has studied the stationary linear problem in the case of a split cylinder rotating at $\Omega_{0}$. Using an asymptotical method, he showed that the flow generated by a slight differential rotation of one part of the shell is composed of two nested cylindrical shear layers aligned with the axis of rotation and located at the split radius. A large geostrophic shear layer of width scaling like $E^{1 / 4}$ (where $E=\nu / \Omega_{0} R^{2}$ is the Ekman 
number, $\nu$ the kinematic viscosity of the fluid and $R$, a characteristic length of the system) accounts for the discontinuity of azimuthal velocity; but a thinner one of size $E^{1 / 3}$, is also necessary to remove the vorticity singularity. Stewartson (1966) solved the similar problem in a spherical shell where the differential rotation is imposed by the central inner core Proudman (1956). Even though the results are slightly more complex, there is still a nested structure of two asymptotic shear layers located at the tangent cylinder (virtual cylinder aligned with the rotation axis and touching the equator of the inner core). The general structure of these detached shear layers was studied by Moore \& Saffman (1969), focusing on the singularities of the boundary-layer equations. Later, a first numerical study in a spherical shell performed by Hollerbach (1994) was complemented by Dormy et al. (1998), recovering the features and scalings predicted by Stewartson.

The linear stability of the Stewartson layers has been studied experimentally by Hide \& Titman (1967). Their set-up was made of a flat cylindrical tank rotating at $\Omega_{0}$ with a central flat disk rotating differentially at $\Delta \Omega$. They found a critical Rossby number ( $\left.R o=\Delta \Omega / \Omega_{0}\right)$ at which the Stewartson layer becomes instable forming geostrophic vortices within the shear layer. They also observed a strong asymmetry between positive and negative differential rotation, unexpected results from the linear point of view which are not yet well understood.

Busse (1968) performed a linear stability theory to the pressure equation of the Stewartson layer. He neglected the bulk viscosity but kept the Ekman friction at the boundary. He recovered some kind of Rayleigh criterion and proposed, for the flat case, a critical Rossby number law : $R o_{c} \sim E^{3 / 4}$. The threshold depends only of the local $R e$, defined for the shear layer as $R e=\Delta \Omega R E^{1 / 4} R / \nu=R o E^{-3 / 4}$.

Later, Niino \& Misawa (1984) performed a new experiment in a flat cylinder but with a disk rotating differentially at the bottom of their tank. Varying the experimental parameters, they found that the destabilisation of the flow is explained by a critical local Reynolds number in agreement with the theory of Busse (1968). They develop a linear quasigeostrophic model to compute the onset of the shear instability. In order to explain accurately the experimental results, they needed to add the bulk viscosity in their model. With this improvement, the quasigeostrophic linear stability analysis is in very good agreement with their experimental results.

In the past few years, Früh \& Read (1999) built an experiment which reproduces almost exactly the case studied by Stewartson (1957): a flat cylinder with two corotating disks at the top and bottom of the tank. Even if the onset is still governed by a critical local Reynolds number, the linear theory of Niino \& Misawa predicts a value for the onset almost two times lower than the one determined in the experiment. They also observed that the behavior of the flow does not depend strongly on the sign of the differential rotation, in discrepancy with the results of Hide \& Titman (1967).

The shear-flow instabilities in a parabolic tank with a free surface was investigated by van de Konijnenberg et al. (1999). They studied both cases with constant depth or varying depth and pointed out that the varying depth has a stabilizing effect, as predicted by the linear theory. However, their setup uses the centrifugal force to adjust the slope of the free surface and consequently the slope is not independent of the rotation rate.

For a varying depth container, a few results exist. Busse (1968) discussed a criterion obtained by Kuo (1949). In the context of oceanography, Kuo (1949) studied the destabilisation of a shear layer with a non-constant depth. Applying his criterion to the Stewartson layer leads to the scaling proposed in this paper (4).

More recently, using a 3D spherical shell code, Hollerbach (2003) studied the differences between positive and negative differential rotation for the onset of these instabilities. By numerical tests, he pointed out the importance of the geometry of the container. A 
second paper by Hollerbach et al. (2004) shows supercritical mode transition in a weakly non-linear regime and compares numerical and experimental results for Ekman number between $10^{-2.5}$ and $10^{-3.5}$ with good agreement.

The study of Dormy et al. (1998) showed that the asymptotic regime of the Stewartson problem cannot be illustrated numerically for Ekman numbers higher than $10^{-5}$. It is the direct result of the asymptotic width of the Stewartson layer $\left(E^{1 / 4} R\right)$ which can appear to be detached only if the Ekman number is much smaller than $10^{-4}$. Consequently, the full 3D calculations and some experiments are too viscous to generalize their results. Their relevance to geophysical flow, where Ekman number are very often lower than $10^{-8}$ is thus limited.

To study the very low Ekman number regimes, we introduce a quasigeostrophic (QG) model able to handle strong $\beta$-effect $(\S 2)$. We compute the axisymmetric basic state flow (Stewartson layer) in $\S 3$. Then, the onset of instability is investigated theoretically to obtain asymptotic laws $(\S 4)$ and numerically to look at the details $(\S 5)$. In $\S 6$, we compare our predictions to various experiments and present new experimental results for spherical geometries. Finally, we discuss our findings in $\S 7$ and the remaining problems that are still to be solved.

\section{Quasigeostrophic model}

Let's consider a container, filled with a fluid of kinematic viscosity $\nu$, axisymmetric around an axis $z$ and rotating at the angular velocity $\Omega_{0}$ around the same axis. We define the cylindrical coordinate system $\left(\boldsymbol{e}_{\boldsymbol{r}}, \boldsymbol{e}_{\boldsymbol{\phi}}, \boldsymbol{e}_{\boldsymbol{z}}\right)$, with $r$ the distance to the axis, $\phi$ the angle in the plane perpendicular to that axis, and $z$ the height. For simplicity, we consider a symmetric container defined by the function $z= \pm L(r)$.

In the frame rotating at angular velocity $\Omega_{0}$, the flow is described by the Navier-Stokes equation. Using $\Omega_{0}{ }^{-1}$ as the time scale and $R$ the radial size of the container as length scale, the dimensionless vorticity equation reads (Greenspan 1968):

$$
\frac{\partial \boldsymbol{\omega}}{\partial t}+(\boldsymbol{\omega} . \nabla) \boldsymbol{u}+(\boldsymbol{u} . \nabla) \boldsymbol{\omega}-2 \frac{\partial \boldsymbol{u}}{\partial z}=E \nabla^{2} \boldsymbol{\omega},
$$

where $\boldsymbol{u}$ is the fluid velocity field, $\boldsymbol{\omega}$ is the vorticity field and $E \equiv \nu / \Omega_{0} R^{2}$ is the Ekman number.

The container is split at the radius $r_{0}$ (see fig. 1). The inner part is differentially rotating at the dimensionless angular velocity $R o=\Delta \Omega / \Omega$ which is a measure of the external forcing on the flow.

For vanishing Ekman and Rossby number, the Proudman-Taylor theorem ensures that the flow is invariant along the rotation axis direction $\left(\frac{\partial \boldsymbol{u}}{\partial z}=0\right)$. For small Rossby and Ekman numbers, the $z$-variations should be weak and the quasigeostrophic (QG) approach assumes a mainly two-dimensional flow for long-period motions $\left(\gg \Omega_{0}^{-1}\right.$ ) (Greenspan 1968).

We z-average $(\langle\rangle)$ the $z$-component of the equation 2.1 with the additional constraint that $u_{r}$ and $u_{\phi}$ are independent of $z$. The $z$-component of the vorticity $\boldsymbol{\omega}$ is denoted by $\omega$ and is also $z$-invariant. We obtain

$$
\frac{\partial \omega}{\partial t}+\left(u_{r} \frac{\partial \omega}{\partial r}+\frac{u_{\phi}}{r} \frac{\partial \omega}{\partial \phi}\right)-(2+\omega)\left\langle\frac{\partial u_{z}}{\partial z}\right\rangle=E \nabla^{2} \omega
$$

where a vortex stretching term $\left\langle\frac{\partial u_{z}}{\partial z}\right\rangle$ is still present.

From the mass conservation, as $u_{r}$ and $u_{\phi}$ are $z$-invariant, we deduce that $u_{z}$ depends linearly on $z$. Then, the stretching term, is $u_{z}^{b} / L$, where $u_{z}^{b}$ is the z-component of the 
velocity at the top and bottom boundary which are the same for symmetric containers (however, the model can be generalized to nonsymmetric cases (Schaeffer 2004))

The non penetration boundary condition gives a first contribution to $u_{z}^{b}$, known as the $\beta$ effect (Pedlosky 1987), and we get $u_{z}^{b}=\beta L u_{r}$ (where $\beta \equiv \frac{1}{L} \frac{\partial L}{\partial r}$ ). A second contribution is given by the pumping induced by the mass conservation in the Ekman layer (away from the $E^{2 / 5}$ equatorial singularity). Greenspan (1968) gave a generic asymptotic expression for this suction and the stretching term then reads :

$$
\frac{\partial u_{z}}{\partial z}=E^{1 / 2} \cdot P\left(u_{r}, u_{\phi}, r\right)+\beta u_{r},
$$

where $P$ is a function of the geometry of the container and linear with respect to the jump of $u_{r}$ and $u_{\phi}$ at the boundary and to their first order derivatives (see Appendix A for the explicit expression for a spherical container).

In the case of a constant-depth container $(\beta=0)$, equation 2.3 reduces to

$$
\frac{\partial u_{z}}{\partial z}=-\frac{E^{1 / 2}}{2 L}\left(\omega-\omega_{b}\right)
$$

where $\omega_{b}$ is the vorticity at the boundary.

In the case of vanishing Ekman number, the Ekman pumping could be neglected compared to the $\beta$ term (see $\S$ C.2). However, the Ekman friction is a dissipative term whereas the $\beta$-term is not. The former is thus expected to play an important role for the non-linear dynamics of quasigeostrophic flows.

At $r=1$, a no-slip boundary condition $u_{r}=u_{\phi}=0$ is used, that avoids any problems at the equator.

We now introduce a scalar field $\psi$, defined by

$$
u_{r} \equiv \frac{1}{r} \frac{\partial \psi}{\partial \phi}
$$

$u_{\phi}$ is now constrained by the three-dimensional mass conservation equation, so that for non-axisymmetric flows,

$$
\begin{aligned}
& u_{\phi}=-\frac{\partial \psi}{\partial r}-\beta \psi \\
& \omega=-\nabla^{2} \psi-\beta\left(\frac{\partial \psi}{\partial r}+\frac{\psi}{r}\right)-\frac{d \beta}{d r} \psi
\end{aligned}
$$

With these equations, we can see that the $\beta$-terms are negligible for $\beta \lambda \ll 1$, where $\lambda$ is a typical radial length scale of the velocity field variations. The model then reduces to the small slope approximation (Busse 1970; Cardin \& Olson 1994; Kiss 2003).

This pseudo-stream function $\psi$ enlarges the use of quasigeostrophic model to domain where the small slope approximation is not valid. Similar approaches have been developed recently. Kiss (2003) proposed a formulation for ocean modeling but without the mass conservation correction. Zavala Sanson \& van Heijst (2002) include the effect of weak topography (small $\beta$ ) and Ekman friction.

For the axisymmetric flow, the scalar function formalism cannot apply. As it is simpler to work with the velocity field, we project the Navier-Stokes equation on $\boldsymbol{e}_{\boldsymbol{\phi}}$. Averaging over $\phi$ to keep only the axisymmetric component, we obtain

$$
\frac{\partial \bar{u}_{\phi}}{\partial t}+\overline{u_{r} \frac{\partial u_{\phi}}{\partial r}}+\frac{\overline{u_{\phi} u_{r}}}{r}+2 \bar{u}_{r}=E\left(\nabla^{2} \bar{u}_{\phi}-\frac{\bar{u}_{\phi}}{r^{2}}\right)
$$

where $\bar{u}$ stands for the $\phi$-average of $u$. 


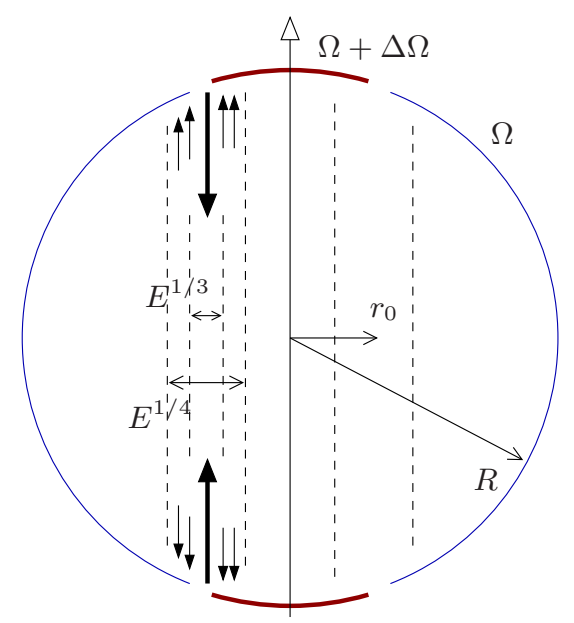

FiguRE 1. Sketch of the nested Stewartson layers in the split-sphere geometry this paper focuses on. The bold arrows show the Ekman pumping inside these layers.

The mass conservation equation reduces to

$$
\frac{1}{r} \frac{\partial r \bar{u}_{r}}{\partial r}+\frac{\partial \bar{u}_{z}}{\partial z}=0
$$

and using equation 2.3, we can show that $\bar{u}_{r}$ is only due to the Ekman pumping and is directly related to $\bar{u}_{\phi}$ :

$$
\bar{u}_{r}=\frac{E^{1 / 2}}{2 L}\left(1+\left(\frac{d L}{d r}\right)^{2}\right)^{1 / 4}\left(\bar{u}_{\phi}-u_{b}\right),
$$

where $u_{b}$ stands for the velocity of the material boundaries (top and bottom).

We solve the system of equations $(2.2,2.3,2.5,2.6,2.7,2.8,2.9)$ numerically to follow the time evolution of $\bar{u}_{\phi}(r)$ and $\psi(r, \phi)$. We use a finite difference scheme in the radial direction, and a Fourier expansion in azimuth. The time integration is done using the semi-implicit Cranck Nicholson scheme for the linear part, and a simple Adams-Bashforth scheme for the non-linear terms, which are computed in direct space, as in Aubert et al. (2003). Provided with the appropriate $\beta$ and $P$ functions, the numerical implementation is able to handle various geometries from flat containers to spherical ones.

\section{Stewartson layers}

Differential rotation in a spherical shell generates the nested Stewartson layers as shown in figure 1 (Stewartson 1966). As our quasigeostrophic model cannot handle discontinuous $\beta$ function, we model the spherical shell geometry by the split sphere geometry following the ideas of Stewartson (1957) for the cylindrical geometry. In the linear and stationary regime, when the Rossby number is small equation 2.8 reduces to a balance between the Ekman pumping term 2.9 and the viscous forces in the bulk.

$$
2 E^{1 / 2} \frac{\alpha}{2 L}\left(\bar{u}_{\phi}-u_{b}\right)=E\left(\nabla^{2} \bar{u}_{\phi}-\frac{\bar{u}_{\phi}}{r^{2}}\right)
$$

which defines the Stewartson layer in our QG model. The asymptotic scaling of width $\Delta \sim$ $E^{1 / 4}$ of the outer Stewartson shear layer is easily recovered. Because of its ageostrophy, 

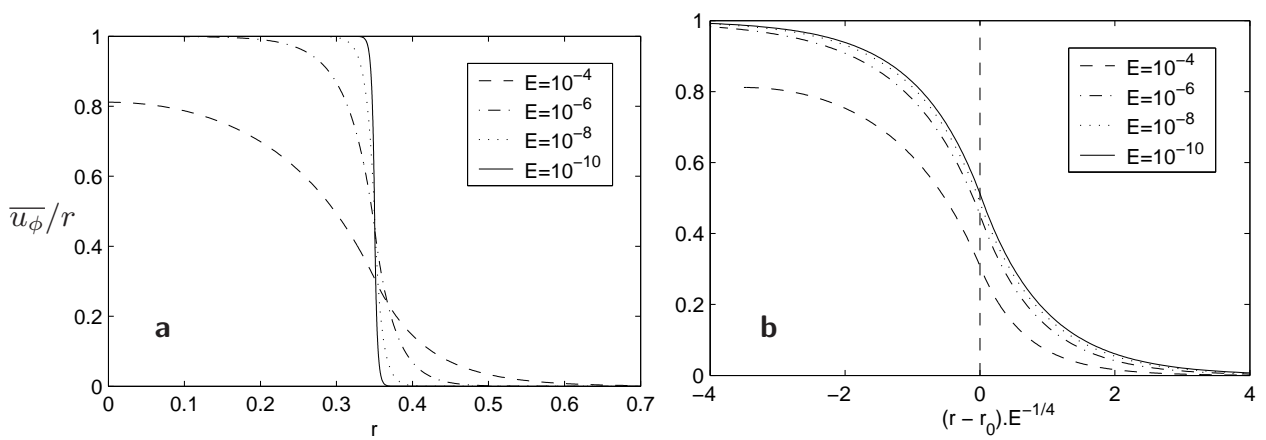

Figure 2. Basic state flow for $r_{0}=0.35$ in the split-sphere geometry. (a) shows the radial profiles and (b) the radial profiles rescaled by the asymptotic size $\left(E^{1 / 4}\right)$ of the Stewartson layer.

the inner $E^{1 / 3}$ shear layer cannot be modeled by a $\mathrm{QG}$ model. However, according to the numerical study of Dormy et al. (1998), the $E^{1 / 3}$-layer has a negligible effect on the velocity profile at Ekman numbers smaller than $E=10^{-6}$.

We compute the basic state flow by solving the linear equation 3.1 for different Ekman numbers. A finite difference radial scheme with variable grid spacing was used for the smallest Ekman numbers. The radial profiles of the average azimuthal velocity are shown in figure 2. Properly scaled by $E^{1 / 4}$, all these profiles superimpose in the asymptotic regime for Ekman numbers lower than $10^{-6}$.

\section{QG asymptotic approach of the onset}

Let $U(r) \boldsymbol{e}_{\boldsymbol{\phi}}$ be the basic state velocity profile solution of equation 3.1, $\Omega=\frac{\partial U}{\partial r}+\frac{U}{r}$ the basic state vorticity. The amplitude of the differential rotation $R o$ imposes the amplitude of the basic flow. Let $\boldsymbol{u}$ (with associated vorticity $\omega$ ) be a perturbed flow of frequency $f$ and with an azimuthal wave-number $m$. The linearised equation 2.2 reads:

$$
\frac{\partial \omega}{\partial t}+R o\left(u_{r} \frac{\partial \Omega}{\partial r}+\frac{U}{r} \frac{\partial \omega}{\partial \phi}\right)-2\left\langle\frac{\partial u_{z}}{\partial z}\right\rangle=E \nabla^{2} \omega
$$

At the stability threshold, we expect the azimuthal size of the perturbation to be comparable to the thickness of the shear layer, so that for the wave-number $m$ we may write $m \sim \Delta^{-1} \sim E^{-1 / 4}$

When there is no slope, the stretching term is given by equation 2.4 and equation 4.1 gives in terms of order of magnitude :

$$
m f+R o \Delta^{-2}+R o m^{2} \sim \frac{E^{1 / 2}}{L} m+E m^{3}
$$

With $m \sim E^{-1 / 4}$, the stability threshold is given by a balance between the inertial forcing and the viscous damping :

$$
R o_{c} \sim E^{3 / 4}
$$

which is the result already obtained by the linear theories of Busse (1968); Niino \& Misawa (1984) corresponding to a stability criteria based on local Reynolds number $\left(R e \sim R o E^{-3 / 4}\right)$ (Niino \& Misawa 1984). For an oscillatory instability, we find the frequency $f \sim E^{1 / 2}$. This time dependence, if present, has not been reported in the previous experiments (Niino \& Misawa 1984; Früh \& Read 1999) and may be very difficult to separate from the advection of the pattern of similar amplitudes. 

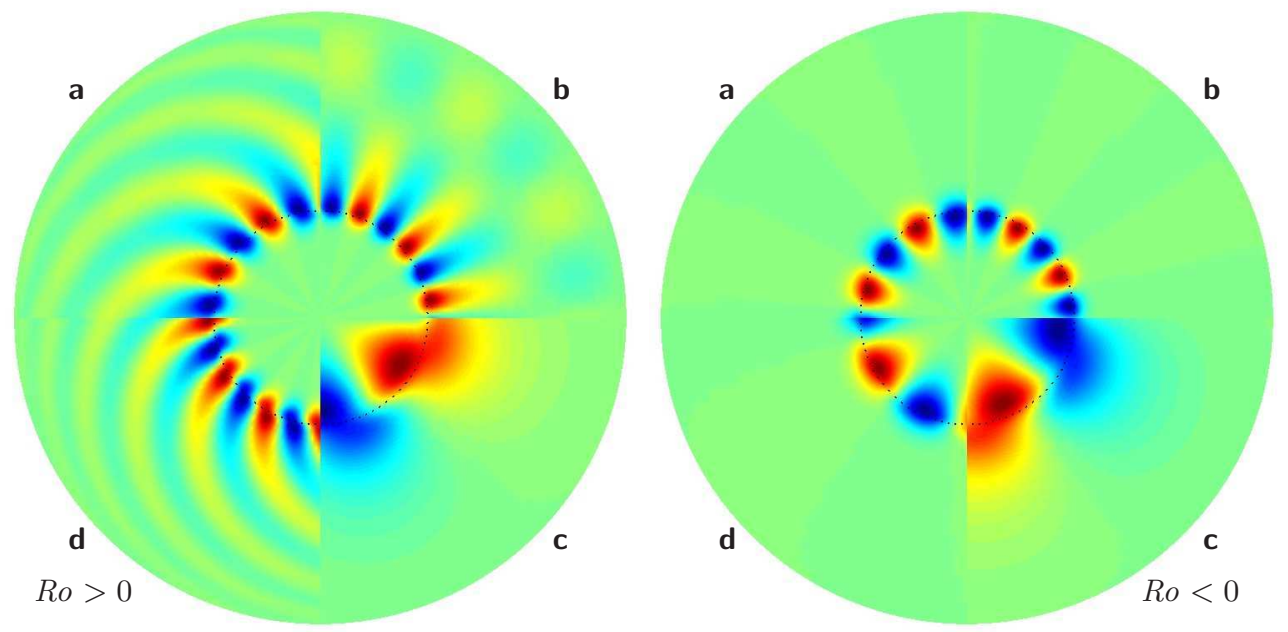

Figure 3. North-pole views of the radial velocity for the first unstable mode at $E=10^{-6}$ for various geometries. Red is positive, blue negative, and green zero. The picture on the left is for $R o>0$, and the one on the right is for $R_{0}<0$. The split-radius $\left(r_{0}=0.35\right)$ is shown by a dotted circle. (a) shows the split-sphere case; (b) shows the constant $\beta$ case $(\beta=-1)$; (c) shows the flat container case $(\beta=0)$; (d) shows the spherical case with flat disks.

When the slope is important enough for the Ekman suction to be small compared to the $\beta$-effect (equation 2.3), equation 4.1 gives the following balance :

$$
m f+R o \Delta^{-2}+R o m^{2}+2 \beta \sim E m^{3}
$$

As $m \sim E^{-1 / 4}$, the viscous term is negligible in front of the $\beta$ term. Consequently, the balance between $\beta$-effect and inertial transport gives :

$$
R o_{c} \sim \beta E^{1 / 2}
$$

This result agrees with the Kuo (1949) criterion applied to a Stewartson layer. For a timedependent instability, the frequency $f \sim \frac{2 \beta}{m}$ follows a Rossby wave dispersion equation.

\section{Numerical calculations of the Stewartson instabilities}

Using the QG-model, for a given Ekman number E, we are able to compute the onset of the instabilities. The Rossby number is increased until a non azimuthal mode grows. The unstable mode for a sphere split at $r_{0}=0.35$ with $E=10^{-6}$ is shown in figure 3a. The Rossby wave instability is mostly concentrated near the Stewartson layer and drifts in the prograde direction as predicted by the dispersion equation ( $\beta$ is negative here) (Pedlosky 1987).

We compute the critical parameters for different Ekman numbers $\left(10^{-5}>E>10^{-10}\right)$. They are given in appendix C.1 and plotted in figure 4. Their Ekman number dependence is in agreement with the asymptotic scaling (4.2) especially for very low Ekman number.

However, significant differences are observed between positive and negative Rossby numbers. The main one is seen in figure $4 \mathrm{c}$ where the critical frequencies differ even at very low Ekman number. Looking at figure 3a, the instability seems to be different as $R o$ changes its sign. When $R o>0(R o<0)$, the critical Rossby wave develops mainly outside (inside) the split radius $r_{0}$. In the inner region, the differential rotation imposes a mean flow which can be considered as solid body rotation for small Ekman numbers. Consequently, we need to subtract the advection of the Rossby wave from the measured 

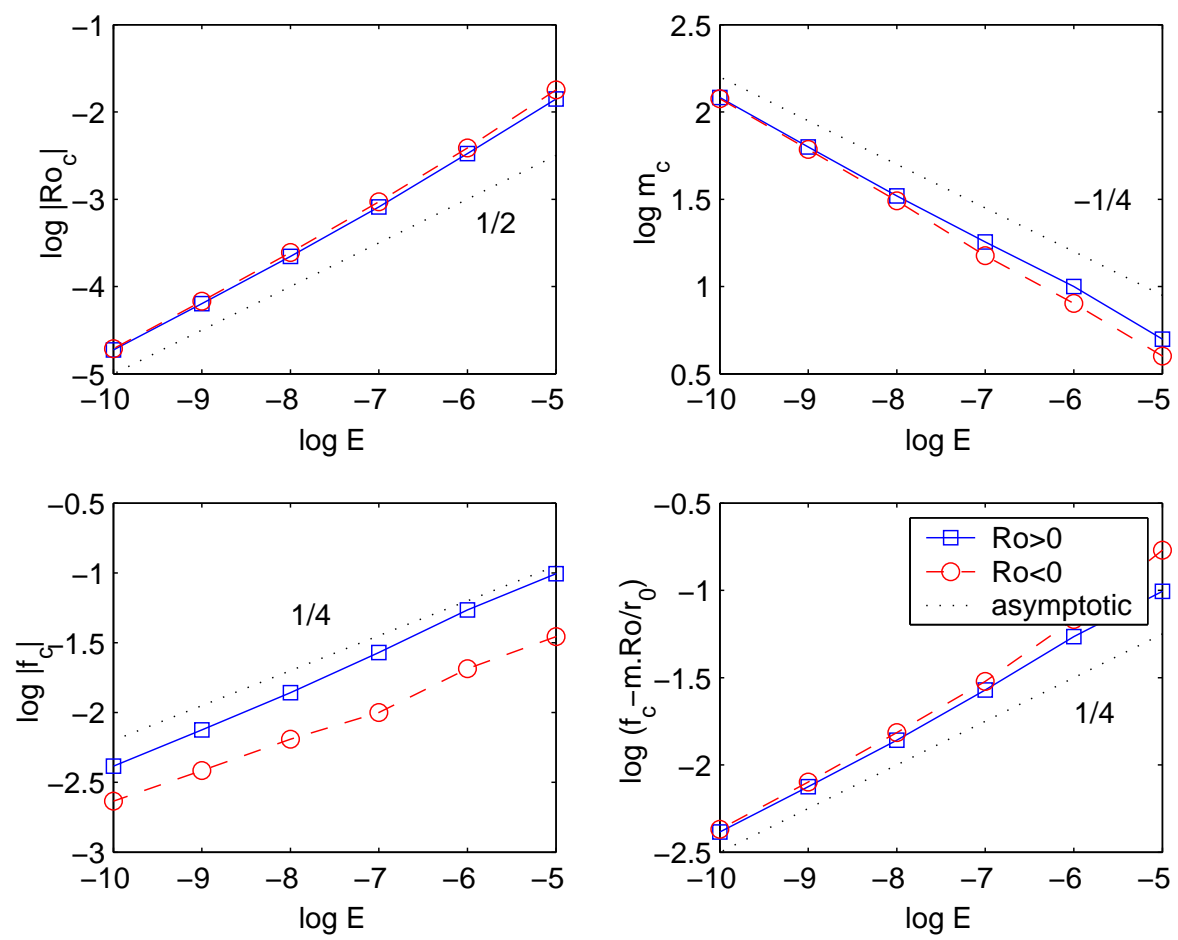

Figure 4. Stability threshold data (given in table C.1) as a function of the Ekman number. (a) represents the threshold; (b) shows the critical wave number; (c) is the raw frequency $f$ at the threshold; (d) is the frequency corrected for advection effects. Dotted lines indicate the asymptotic behavior.

critical frequency $f-2 \pi m R o$ for $R o<0$ while no correction is needed for $R o>0$. The corrected frequencies of the Rossby waves are plotted in figure $4 \mathrm{~d}$, and both positive and negative $R o$ agree and follow the predicted asymptotic law $E^{1 / 4}$.

Another small difference could be noticed between positive and negative critical Rossby numbers ( $3 \%$ for $E=10^{-10}$ ), which could be due to the variations of $\beta$ along the radius. To test this idea, the QG model was used to compute the onset of instability for constant $\beta=-1$ (corresponding to a container with an exponential shape). The instability is a Rossby wave (figure $3 \mathrm{~b}$ ) with the expected asymptotic exponents but we still find a comparable difference between positive and negative differential rotation. Noting with $\mathrm{a}+(-)$ exponent the positive (negative) differential rotation values obtained, we have $\left|R o_{c}^{+}\right|<\left|R o_{c}^{-}\right|$and $m_{c}^{+}>m_{c}^{-}$. Hence, we must explain the difference between positive and negative differential rotation by a curvature effect. When the instability develops in the inner region, it has less space, so that the critical $m$ is reduced, implying also a critical Rossby number of larger magnitude. Indeed, Increasing the split-radius $r_{0}$ reduces these differences.

In all cases where $\beta \neq 0$, the instability develops either in the inner region or in the outer part (delimited by the split-radius) depending on the sign of the Rossby number (see fig. 3). We understand this radial dependence using the Rossby wave mechanism (Pedlosky 1987). By conservation of potential vorticity, a negative $\beta$ effect tends to amplify negative (positive) vorticity as a column moves away from the center to shallower (deeper) region. For $R o>0$, the Stewartson shear layer is a negative vorticity sheet implying that the perturbation is damped in the deeper region, whereas it is amplified in 
the shallow region. For $R o<0$, it is the opposite. In both cases, the amplified vorticity will induce a flow which induces a drift of the pattern in the prograde direction (as for any Rossby wave with $\beta<0$ ).

If you consider a flat container $(\beta=0)$, the instability is not a Rossby wave any more but a geostrophic shear instability with a very different signature (figure $3 \mathrm{c}$ ). The expected asymptotic exponents have been retrieved in agreement with Niino \& Misawa (1984) and we found no difference between positive and negative critical Rossby numbers.

The perturbation exhibits spiraling vorticity cells in the split-sphere case while the Rossby wave has a constant phase along the radius when $\beta$ is constant (see fig. 3a and $3 \mathrm{~b}$ ). As already explained in the case of thermal convection in a rapidly rotating sphere, the prograde spiralisation of the Rossby wave is a consequence of the decrease of $\beta$ with increasing $r$ (Zhang 1992; Cardin \& Olson 1994; Jones et al. 2000).

An interesting feature is the radial extent of the Rossby wave. For $R o>0$, the instability develops in the whole outer region (figure $3 \mathrm{a}$ and $3 \mathrm{~b}$ ) and this statement stays true for any Ekman number we have computed. In the flat case (figure 3c), the radial extent of the shear instability decreases as $E^{1 / 4}$, following the width of the Stewartson layer. In the thermal convective case, the critical thermal Rossby wave is localised in a radial domain of width $E^{1 / 6}$ (Yano 1992; Jones et al. 2000; Dormy et al. 2004). On the contrary, the prograde Stewartson instabilities spread all the way to the outer equator. A simple heuristic model, detailed in appendix B, explain this feature as a consequence of the asymptotic exponents of the critical parameters.

As the flow approaches the outer equator, its description in the QG model fails for two reasons: $\beta$ tends to infinity, and the Ekman pumping effect is not valid as the Ekman layer becomes singular (Roberts \& Stewartson 1963). However, this area is very small and we expect that it will not affect the determination of the stability threshold. To test this statement, we followed the idea of Yano et al. (2003) and truncated our container at $r=0.9$ to prevent the slope from growing to large near the equator. The solutions showed the same features as the untruncated calculation and very similar stability thresholds.

With our numerical code, we are able to test various boundary conditions. We report that the boundary conditions (free-slip or no-slip) hardly affect the stability threshold, whereas the presence of Ekman friction has a small yet visible effect. The divergence correction we introduced in equation 2.6 seems to be negligible at the threshold. The results of these tests are summarised in table C.2.

\section{Comparison with experiments}

\subsection{Flat containers}

The constant depth experiments of Niino \& Misawa (1984) are reproduced numerically using our QG model. Results are reported in table 1 and agree surprisingly well with the original experimental data, as did their own numerical calculations.

The QG model is also used to reproduce numerically the experiments done by Früh \& Read (1999). The results of our linear calculation for the threshold are significantly lower than the experimental ones, but are in good agreement with the theory of Niino \& Misawa (1984). Nevertheless, the critical wave-length is in good agreement as shown in table 2. To explain the discrepancy between experiment and theory, Früh \& Read (1999) invoked the lack of the inner $E^{1 / 3}$ layer in the modeling of Niino \& Misawa (1984), which is also missing in this QG-model. 


$\begin{array}{ccccc}E & R o_{c}^{N} & m_{c}^{N} & R o_{c} & m_{c} \\ 3.010^{-5} & 0.025 & 5-6 & 0.024 & 5 \\ 6.010^{-6} & 0.0075 & 7-9 & 0.0072 & 8\end{array}$

TABLE 1. Comparison between the experimental results of Niino \& Misawa (1984) (denoted by .$^{N}$ ) and our numerical results.

$\begin{array}{ccccc}E & R o_{c}^{F} & m_{c}^{F} & R o_{c} & m_{c} \\ 1.110^{-5} & 0.022 & 6-8 & 0.0105 & 6 \\ 8.310^{-6} & 0.016 & 6-8 & 0.0085 & 6 \\ 5.610^{-6} & 0.0125 & 6-8 & 0.0065 & 7\end{array}$

TABLE 2. Comparison between the experimental results of Früh \& Read (1999) (denoted by .$^{F}$ ) and our numerical results.

FiguRE 5. Sketch of the experimental setup with flat disks. The spherical shell geometry is obtained with a central inner sphere instead of the two disks on the shaft (see fig. 6)

\subsection{Varying depth containers}

Figure 5 shows a sketch of the experimental set-up we use to study the onset of instability of the Stewartson layer. It consists of a plexiglass ellipsoid put in rotation by a brushless motor along its minor axis with a controlled angular velocity $\Omega_{0}$. The working fluid is water $\left(\nu=10^{-6} \mathrm{~m}^{2} / \mathrm{s}\right)$. On the axis of rotation a shaft associated to a step motor can drive either a central inner core or two disks. Exact dimensions are reported in fig. 5 and further details can be found in Noir et al. (2003).

To determine the stability threshold, we use a flake solution (Kalliroscope AQ 1000) and illuminate our container with a light sheet in a meridional plane. Variations of brightness allow us to visualize the shear field in the illuminated plane. When the shear field is steady in the laboratory frame, we assume that the flow is stable. When the shear becomes unsteady (brightness oscillations in time in the laboratory frame), we consider the flow unstable. Pictures of the observed patterns are shown on figure 6 , showing that 

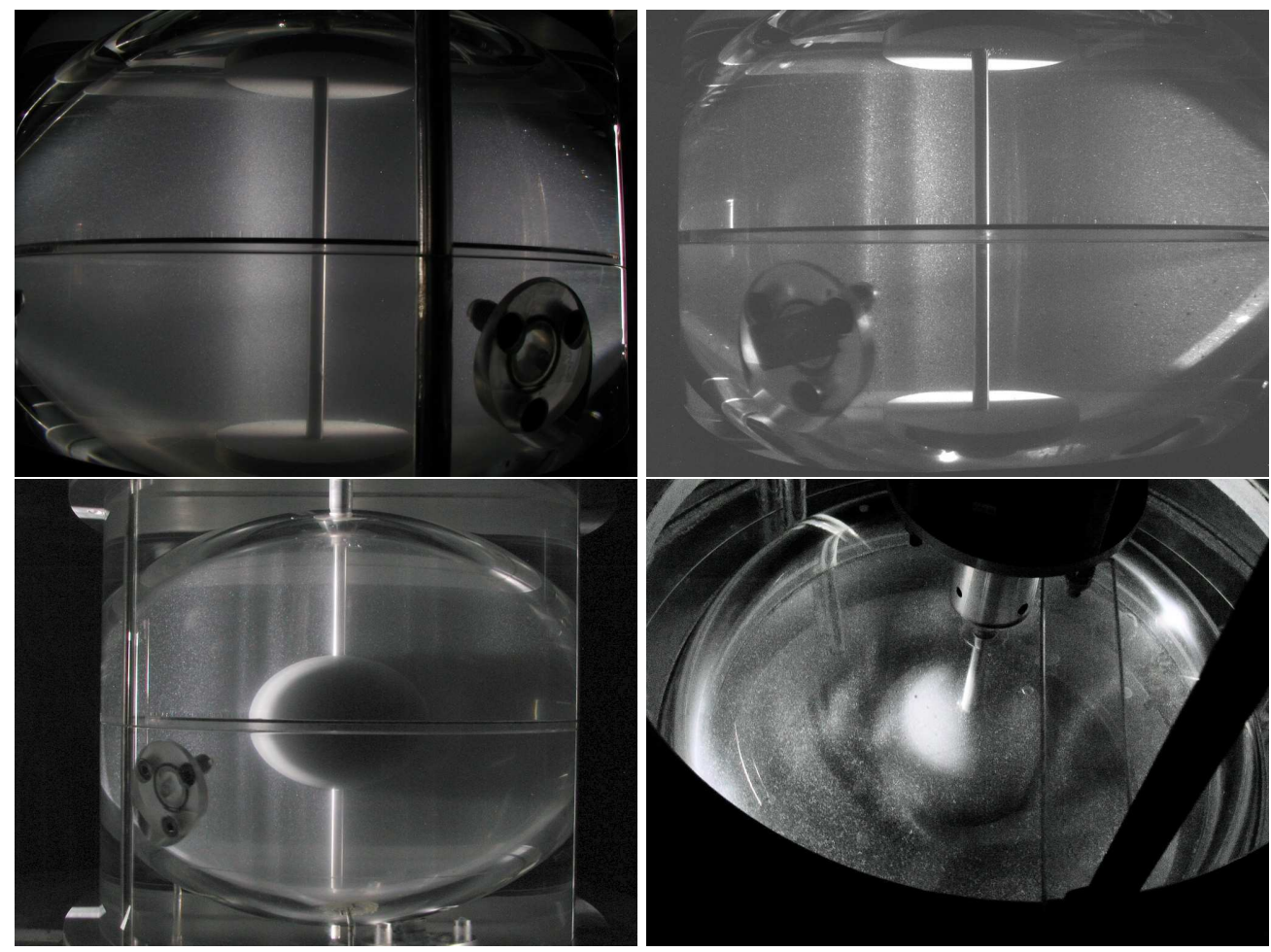

FIGURE 6. Instabilities near the threshold as seen in our experiments at $E \simeq 2 \cdot 10^{-5}$. The shaft holding the disks or inner sphere is along the rotation axis. The features shown by the flakes are aligned with the rotation axis, supporting the QG-approximation for our model. Top left panel shows a stable axisymmetric flow $(R o=0.05)$ driven by two corotating disks, that becomes instable on the top right panel as the disks rotate faster $\left(R_{o}=0.2\right)$. At the bottom, we show the instable flow driven by a rotating inner sphere for $R o>0$, illuminated with a meridional light sheet on the left (equator view), and with a light sheet parallel to the equator on the right (north pole view).

the features of the flow are aligned with the rotation axis. The thin bright and dark columns appear at the onset and are blinking, showing the advection of the pattern of alternating vortices.

With the two disks geometry, the onset is obtained for different rotation rates (from 30 rpm to $200 \mathrm{rpm}$ ) by different persons independently. Results for the stability threshold are summarised in figure 7 and quantitative data are reported in table C.3. The critical Rossby number evolution with $\mathrm{E}$ does not form a clear straight line. It could be due to the integer nature of the critical azimuthal wave number. The data seems to be grossly compatible with the $E^{1 / 2}$ asymptotic scaling. However, with only one decade of data and for such high Ekman numbers, we do not expect the asymptotic laws to hold. Moreover, for $R o<0$, we expect the data to follow an $E^{3 / 4}$ law associated to the flat geometry of the inner part. Unfortunately, this visualisation technique does not enable the measurement of critical frequency and the critical wave number but using a strobe at the frequency of the container, we have been able to see the prograde drift of the Rossby wave.

For negative differential rotation, the onset was very difficult to determine. We had to wait sometimes for hours to be sure of the results. The onset is very sensitive to boundary conditions such as the presence of small air bubbles attached to the tank walls. In some cases, a very low frequency oscillation has been observed $(m=1$ ?) instead of the Rossby 


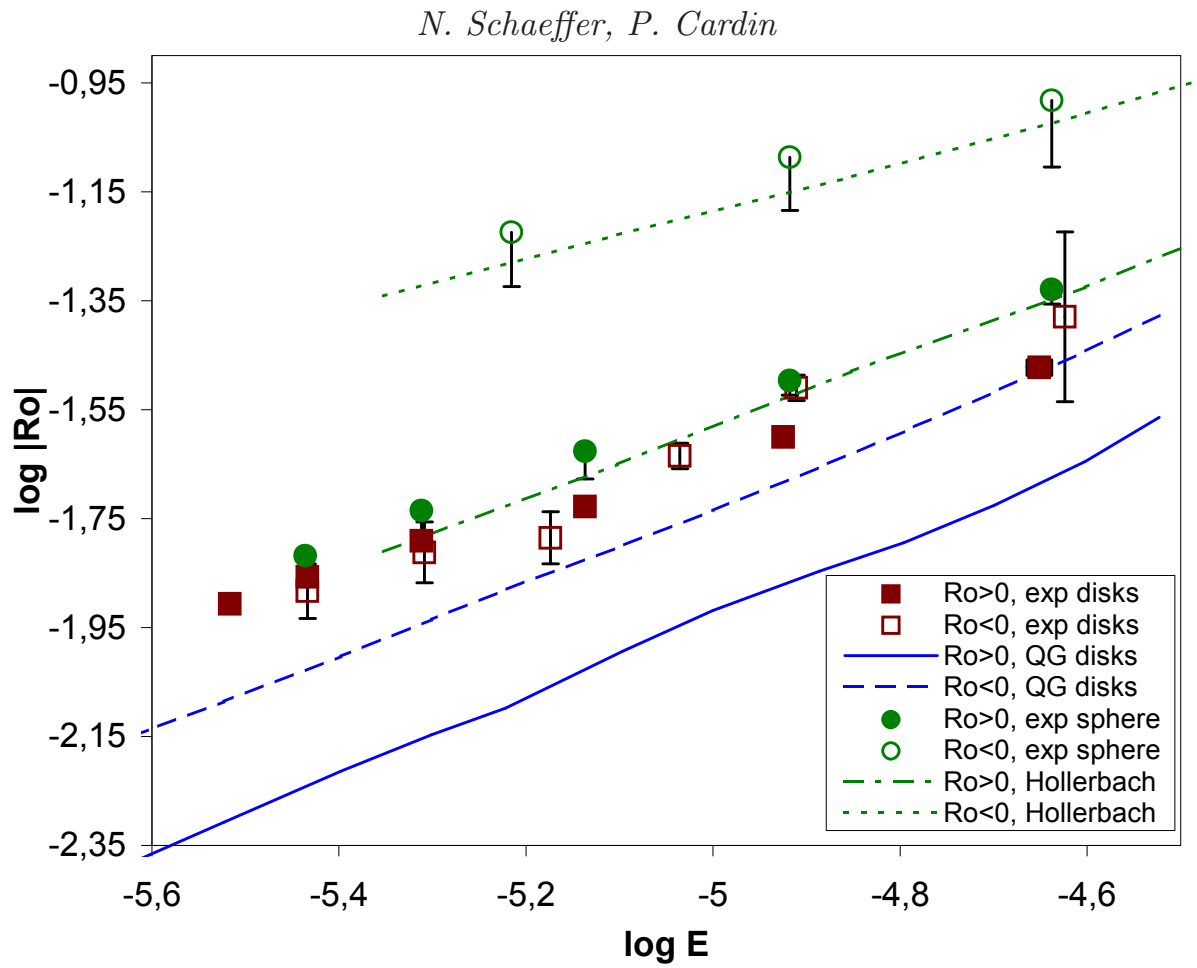

FiguRE 7. Experimental determination of the stability threshold for two geometries, compared with the numerical calculations using a quasigeostrophic model. sphere $3 D$ is computed with a fully $3 \mathrm{D}$ model. The negative differential rotation data are not well defined. The numbers are the slopes of the linear fits.

wave instability but these results were not reproducible and we have no explanation for them.

We use the QG model to reproduce the experiment. The two polar caps of the splitsphere geometry are replaced by two flat disks in order to mimic the experimental set-up. However, the QG-model cannot handle height-jumps at the edge of the disks, so modeled geometry is in fact flat for $r<r_{0}$ and spherical for $r>r_{0}$, with no height jump. The critical Rossby numbers for negative and positive differential rotation are shown in figure 7 and the spatial structure of the instability is represented in figure $3 \mathrm{~d}$. The numerical QG onset is below the experimental determination by almost a factor 2 for $R o<0$ and a factor 3 for $R o>0$. Such a ratio was also noticed in the experiment of Früh \& Read (1999).

\subsection{Spherical shell geometry}

A plastic inner core $(8 \mathrm{~cm}$ of diameter) has been attached to the shaft at the center of the container. The onset has been determined optically under the conditions described above. Quantitative critical Rossby numbers are reported in table C.4 and are plotted in figure 7. Our visual determination of the Rossby wave instability is in good agreement with the 3D numerical calculations of Hollerbach (2003). This makes us confident in the reliability of the optical method to determine the onset.

As in the disks case, the onset was also very difficult to determine for negative Rossby numbers. Nevertheless, the negative differential rotation critical branch lies a factor 3 above the the positive one on both experimental and numerical determination. The case of a spherical shell is quite singular : $\beta$ has opposite sign for $r<r_{0}$ and $r>r_{0}$, and 


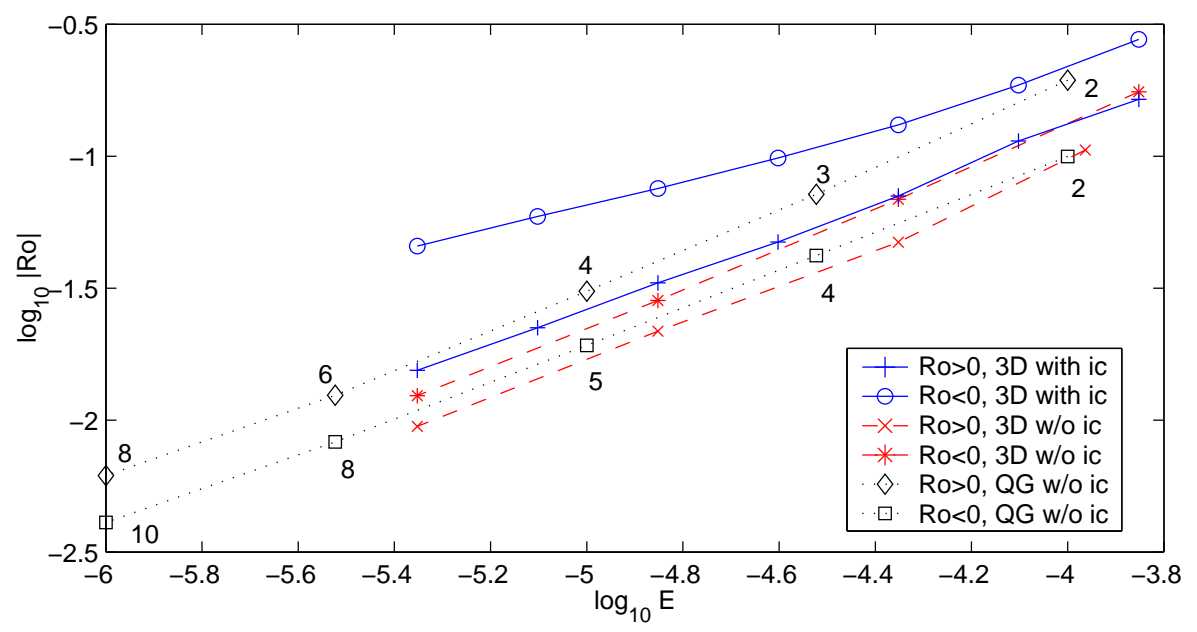

FiguRE 8. Critical Rossby numbers obtained by Hollerbach (2003, summary of his fig. 4 and 8 a, here denoted by $3 D$ ) showing the impact of the slope on the stability threshold. We also plotted the corresponding results obtained with our QG-model (basic state shear layer obtained with an inner core, instabilities computed without) including the critical mode. When removing the inner core (ic), the threshold drops, and positive and negative Rossby numbers get closer. Our QG-model is close to the fully 3D results of Hollerbach

the magnitude of $\beta$ is much higher for $r<r_{0}$. This implies that Rossby waves travel in opposite directions in these two regions. For positive Ro, the instability may develop either in the $r>r_{0}$ region or in the $r<r_{0}$ one. In both directions the depth is decreasing, but from eq. 4.2, we expect that the Rossby wave will appear in the outer region $\left(r>r_{0}\right)$ where the magnitude of $\beta$ is lower and then propagate in the prograde direction. However, for negative $R o$ there is no deeper region around the shear layer, where the instability may be amplified by the $\beta$ effect, so that we expect the threshold to be much higher and the instability to be of a different kind, as the very small azimuthal wave-number observed by Hollerbach (2003) may suggest.

To understand the geometrical effect on the instability, Hollerbach (2003) computed the basic-state shear layer in a spherical shell geometry, then took the velocity profile obtained at mid-depth and extended it to all $z$, generating a $z$-invariant profile in a full sphere without an inner core (see Hollerbach 2003, §5). He finally computed the instabilities of that flow and found critical curves close to the $R o>0$ case. This result may be understood in the following way: by artificially removing the inner sphere, the $\beta$ function becomes monotonic and looses the singular geometrical property described above, so that the two onsets, now controlled by the moderate $\beta$-effect of the outer sphere, become comparable.

We repeated exactly the same procedure with the QG-model : we computed the basic state shear layer with an inner-core $\left(r_{0}=0.35\right)$, and then computed the stability threshold of this velocity profile in a full sphere geometry (without inner core). The results are compared with the 3D computations of Hollerbach on figure 8. For $R o<0$, the QGmodel gives thresholds about $30 \%$ higher, and only $6 \%$ higher for $R o>0$. The critical modes obtained are also in very good agreement. 

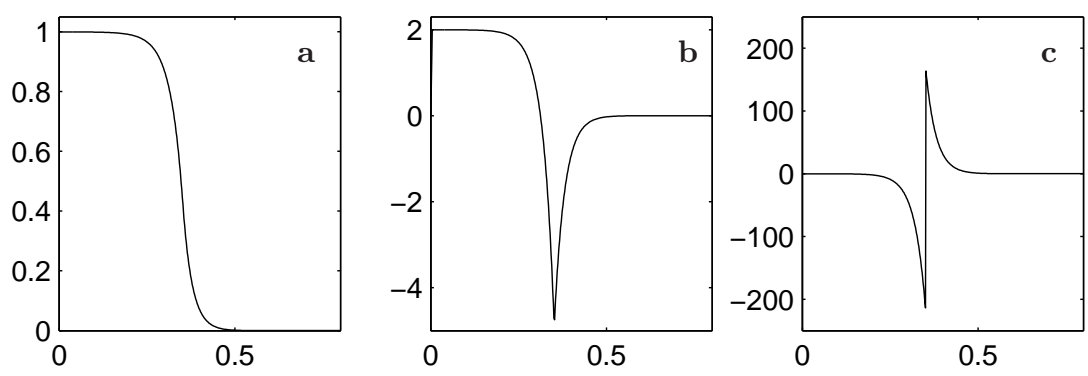

FiguRE 9. Basic state axisymmetric flow profiles (solution of eq. 3.1), as a function of radius $r$ for $E=10^{-6}$ and a split-sphere geometry with $r_{0}=0.35$. (a) rotation velocity $U / r$; (b) vorticity $\Omega ;\left(\right.$ c) radial derivative of vorticity $\frac{\partial \Omega}{\partial r}$.

\section{Discussion and conclusion}

In this study, we give some insight into the instability of a Stewartson shear layer in the very low Ekman regime. The QG-model with the pseudo-stream function formalism is designed for finite slopes as it handles the mass conservation correctly. Despite failing to describe the $E^{1 / 3}$-layer, it has proved to be a valuable tool for numerical experiments to study very low Ekman numbers. It can also be used for non-linear calculations as long as the quasigeostrophic hypothesis stands, and may be thus useful for ocean and planetary interior models.

The model allows us to compare flat top and bottom geometries with varying depth containers. Two different stabilizing processes are at work, leading to different balances and scalings for the threshold : for flat containers the Busse (1968) $E^{3 / 4}$ scaling applies for shear instabilities, whereas with a slope a $\beta E^{1 / 2}$ scaling is predicted associated to a Rossby wave instability.

The quasigeostrophic model of the Stewartson layer gives a critical Rossby number too low in the flat experiment of Früh \& Read (1999) and in our spherical experiment. As proposed by Früh \& Read (1999), the nested $E^{1 / 3}$-layer missing in the QG description may be responsible of this discrepancy. Taking the Rayleigh criterion obtained by Kuo (1949), we may enforce this idea. It says that the critical Rossby number evolves like $R o_{c} \sim \beta / \frac{\partial \Omega}{\partial r}$ where $\Omega$ is the vorticity of the basic state flow. Using the QG model, we plot the basic state profiles in figure 9. We see that $\left|\frac{\partial \Omega}{\partial r}\right|$ is maximum at the split-radius where it is also discontinuous. Stewartson (1957) introduced the $E^{1 / 3}$-layer to smooth out the discontinuity of $E^{1 / 4}$-layer. The presence of the ageostrophic $E^{1 / 3}$-layer reduces the amplitude of $\frac{\partial \Omega}{\partial r}$ and thus increases the onset of excitation of the Rossby wave according to the Kuo (1949) criterion. If this is true, a three dimensional model could be used to determine the Stewartson layer, axisymmetric stationary basic state flow, for very low Ekman numbers Dormy et al. (1998); Hollerbach (2003) and the onset of Rossby wave instability, and its critical parameters, may be computed using a QG model. In any case, it is difficult to imagine that this approach will solve the discrepancy between the flat cylindrical experiments of Früh \& Read (1999) and Niino \& Misawa (1984)

At very low Ekman numbers $E<10^{-6}$, we report an interesting radial structure, comparable to the one exhibited in the thermal instability case by Dormy et al. (2004). However, if the global structure of the flow is quite similar to the spiraling thermal convection flow in a rapidly rotating spherical shell, the radial extension of the instability is surprisingly independent of the Ekman number. Using a simple heuristic model, we propose an explanation based on the asymptotic scalings of the instability.

Hollerbach (2003) studied the difference between positive and negative Rossby num- 
bers, and pointed out that the geometry is the crucial feature, through the radial derivative of the height of the container. The numerical calculations he made shed some light on the dramatic changes in the critical mode when switching from positive to negative Rossby numbers observed in some real experiments (Hide \& Titman 1967). However, ignoring that Rossby waves are excited in varying depth containers, his conclusions for spherical shell cannot be applied to flat containers and the initial experimental observation of Hide \& Titman (1967) remains mysterious.

We demonstrated in this paper that the geometrical effects are due to a fundamental symmetry breaking in theRossby wave propagation mechanism: depending on the sign of $R o$ and $\beta$, the instability develops on one side of the shear layer or the other. This symmetry breaking occurs in any geometry, without the need for abrupt changes in the height of the container (even though this asymmetry will be amplified by such discontinuities). Furthermore, the spherical shell setup is singular: when $R o<0$, the Rossby wave instability cannot develop on either side of the shear layer, leading to the singular results observed in this configuration. Within this framework, the recent results of Hollerbach (2003) can be interpreted correctly.

The true nature of the instability in that particular case (spherical-shell and $R o<0$ ) remains unclear. Is it a Rossby wave that needs much stronger forcing to grow, or another type of instability with significant 3D effects? This uncertainty is annoying since this geometry is of geophysical interest for gaseous planets and planetary interiors. It is also the geometry used in the sodium experiment presented by Cardin et al. (2002), designed to study the magnetic induction processes in this type of flow, which are suspected to be able to sustain a dynamo. In addition, the non-linear and turbulent regimes have to be investigated, by means of numerical calculations and quantitative velocity measurements in experiments. We strongly need experimental and numerical data at lower Ekman numbers, especially in the spherical shells geometry.

\section{Acknowledgements}

Most of the computations presented in this paper were performed at the Service Commun de Calcul Intensif de l'Observatoire de Grenoble (SCCI).

We wish to thank Henri-Claude Nataf for stimulating discussions about this work. We are also grateful to Natalia Bezaeva, Daniel Brito and Jean-Paul Masson for their help in running the experiment.

This work was funded by the program DyETI and PNP of the CNRS/INSU.

\section{Appendix A. Ekman Pumping in a sphere}

The Ekman pumping flow is given by Greenspan (1968)

$$
\boldsymbol{u} .\left.\boldsymbol{n}\right|_{ \pm L}=-\frac{E^{1 / 2}}{2} \boldsymbol{n} . \boldsymbol{\nabla} \times\left(\frac{\boldsymbol{n} \times \boldsymbol{u} \pm \boldsymbol{u}}{\sqrt{\left|\boldsymbol{n} \cdot \boldsymbol{e}_{\boldsymbol{z}}\right|}}\right)
$$

with $\boldsymbol{n}$ the outward unit vector normal to the surface and $\boldsymbol{u}$ the velocity jump between the geostrophic interior and the boundaries. 
We can separate this

$$
-\frac{1}{2} E^{1 / 2}(\underbrace{\boldsymbol{n} . \nabla \times\left(\frac{n \times u}{\sqrt{\left|\boldsymbol{n} \cdot \boldsymbol{e}_{\boldsymbol{z}}\right|}}\right)}_{a} \pm \underbrace{\boldsymbol{n} . \nabla \times\left(\frac{u}{\sqrt{\left|\boldsymbol{n} \cdot \boldsymbol{e}_{\boldsymbol{z}}\right|}}\right)}_{b})
$$

In this appendix, the spherical coordinate system $(\rho, \theta, \phi)$ is used to develop that expression noting that $\boldsymbol{n}=\boldsymbol{e}_{\boldsymbol{\rho}}$. Term $a$ reduces to

$$
a=|\cos \theta|^{-1 / 2}\left( \pm \frac{|\tan \theta|}{2 \rho} u_{\theta}-\frac{\partial u_{\rho}}{\partial \rho}-\frac{2}{\rho} u_{\rho}\right)
$$

and term $b$ can be written as

$$
b=|\cos \theta|^{-1 / 2}\left( \pm \frac{|\tan \theta|}{2 \rho} u_{\phi}+\omega_{\rho}\right) .
$$

On the boundary, we have $\rho=1$, and so

$$
\boldsymbol{u} .\left.\boldsymbol{n}\right|_{ \pm L}=\frac{E^{1 / 2}}{2|\cos \theta|^{1 / 2}}\left(\frac{|\tan \theta|}{2}\left( \pm u_{\theta}-u_{\phi}\right) \pm \omega_{\rho}-2 u_{\rho}-\frac{\partial u_{\rho}}{\partial \rho}\right)
$$

Let's apply the QG approximation described in $\S 2$. We need to write A 2 into cylindrical coordinate system $(r, \phi, z)$. We have

$$
\begin{aligned}
& \rho \sin \theta=r, \rho \cos \theta=z, \\
& u_{\theta}=u_{r} \cos \theta-u_{z} \sin \theta, u_{\rho}=u_{r} \sin \theta+u_{z} \cos \theta, \\
& \frac{\partial}{\partial \rho}=\frac{\partial r}{\partial \rho} \frac{\partial}{\partial r}+\frac{\partial z}{\partial \rho} \frac{\partial}{\partial z}=\sin \theta \frac{\partial}{\partial r}+\cos \theta \frac{\partial}{\partial z} .
\end{aligned}
$$

Furthermore, $u_{r}$ and $u_{\phi}$ are $z$-independent, and $u_{z}$ is a linear function of $z$ :

$$
u_{z}=z \frac{-r}{1-r^{2}} u_{r}(r, \phi) \text {. }
$$

We have

$$
\frac{\partial u_{\rho}}{\partial \rho}=\frac{-2 r}{1-r^{2}} u_{r}
$$

and

$$
\omega_{\rho}=\omega_{z} \cos \theta+\omega_{r} \sin \theta
$$

where $\omega_{r}$ is given by

$$
\omega_{r}=\frac{1}{r} \frac{\partial u_{z}}{\partial \phi}=\frac{-z}{1-r^{2}} \frac{\partial u_{r}}{\partial \phi}
$$

At the end, we get:

$$
\begin{aligned}
\boldsymbol{u} .\left.\boldsymbol{n}\right|_{ \pm L}= & \frac{E^{1 / 2}}{2\left(1-r^{2}\right)^{1 / 4}}\left(\frac{-r}{2\left(1-r^{2}\right)^{1 / 2}} u_{\phi}+\frac{5}{2} \frac{r}{1-r^{2}} u_{r}\right. \\
& \left.-\left(1-r^{2}\right)^{1 / 2}\left(\omega_{z}-\frac{r}{1-r^{2}} \frac{\partial u_{r}}{\partial \phi}\right)\right)
\end{aligned}
$$

This expression can be treated just like the no-slip boundary condition (Pedlosky 1987):

$$
\left.\boldsymbol{u . n}\right|_{ \pm L}=r u_{r} \pm \sqrt{1-r^{2}} u_{z}
$$


from which we deduce $u_{z}$ at the top and bottom boundary

$$
\pm\left. u_{z}\right|_{ \pm L}=\left(1-r^{2}\right)^{-1 / 2}\left(\boldsymbol{u} .\left.\boldsymbol{n}\right|_{ \pm L}-r u_{r}\right) .
$$

From the linear $z$-dependence of $u_{z}$ we obtain the stretching term :

$$
\begin{aligned}
\frac{\partial u_{z}}{\partial z}= & \frac{E^{1 / 2}}{2\left(1-r^{2}\right)^{3 / 4}}\left(-\omega_{z}+\frac{r}{1-r^{2}}\left(\frac{\partial u_{r}}{\partial \phi}-\frac{1}{2} u_{\phi}\right)-\frac{5 r}{2\left(1-r^{2}\right)^{3 / 2}} u_{r}\right) \\
& +\frac{-r}{1-r^{2}} u_{r} \\
= & E^{1 / 2} \cdot P\left(u_{r}, u_{\phi}, r\right)+\beta u_{r}
\end{aligned}
$$

\section{Appendix B. A simple model for the radial scaling}

\section{B.1. Approximations and assumptions}

In figure $3 a b c$ the perturbation of the shear layer spreads across the whole volume whereas in the flat container case (fig. 3d) or even the thermal convection case in a sphere (see Dormy et al. 2004), it is radially localised. In this section we derive a simple heuristic model that predict all these properties outside the Stewartson shear layer.

To simplify our study, we make a few approximations :

- we neglect the curvature;

- we drop the viscous dissipation term, but keep the Ekman friction;

- we assume small forcing, ignoring the local vorticity term.

The curvature may be negligible if the scales involved are small enough. Looking at the results from section $\S 4$, we expect the scales to shrink while lowering the Ekman number. Thus, we replace the angular variable $\phi$ by $y$. Dropping the viscous term lowers the order of the differential equation and makes it easier to handle. Numerical simulations with no viscous term or an artificially weakened viscous term show that the structure stays very similar. In addition, the threshold is expected to be only slightly affected in the asymptotic regime. This is because the Ekman friction is also a dissipative term. Finally, we can neglect the local vorticity term because in the linear stability case it is a higher order effect, as shown in section 4 . We end up with

$$
\frac{\partial \omega}{\partial t}+R o\left(U \frac{\partial \omega}{\partial y}+u_{r} \frac{\partial \Omega}{\partial r}\right)=2\left\langle\frac{\partial u_{z}}{\partial z}\right\rangle
$$

The expression of the Ekman friction involves several terms. We will only keep the vorticity contribution, so that

$$
\left\langle\frac{\partial u_{z}}{\partial z}\right\rangle \simeq \beta u_{r}-\alpha E^{1 / 2} \omega
$$

where $\alpha$ is the geometrical factor for the Ekman friction term, and for a sphere $\alpha=$ $1 / 2\left(1-r^{2}\right)^{-3 / 4}$ (see eq. A 6 ).

We now assume a perturbation of the form

$$
\Psi(r, y, t)=\psi(r) e^{i(k y-\omega t)}
$$

and we obtain

$$
\left(-i \omega+R o U i k+E^{1 / 2} \alpha\right)\left(k^{2}-\frac{\partial^{2}}{\partial r^{2}}\right) \psi+\left(R o \frac{\partial \Omega}{\partial r}-2 \beta\right) i k \psi=0
$$


In a more convenient form, we can write

$$
\frac{\partial^{2} \psi}{\partial r^{2}}=k^{2}\left(1+\frac{1}{k} \frac{\left(2 \beta-R o \frac{\partial \Omega}{\partial r}\right)\left(\omega-R o U k-i E^{1 / 2} \alpha\right)}{(\omega-R o U k)^{2}+E \alpha^{2}}\right) \psi
$$

\section{B.2. Outer part, large $\beta$}

In the outer part the basic state velocity profile $U$ is vanishing, as well as $\frac{\partial \Omega}{\partial r}$. This simplifies the previously established relation B 4 :

$$
\frac{\partial^{2} \psi}{\partial r^{2}}=k^{2}\left(1+\frac{2 \beta}{k} \frac{\omega-i E^{1 / 2} \alpha}{\omega^{2}+E \alpha^{2}}\right) \psi
$$

In the case of small Ekman number, $E \alpha^{2}$ is negligible compared to $\omega^{2} \sim E^{1 / 2}$, but we have to keep the small imaginary part. We now set

$$
\gamma=k\left|1+\frac{2 \beta}{k \omega}\right|^{1 / 2}
$$

If $|2 \beta| \gg 1$, we can rewrite B 5 as

$$
\frac{\partial^{2} \psi}{\partial r^{2}}=-\gamma^{2}\left(1-\frac{2 i \beta E^{1 / 2} \alpha}{k \omega^{2}+2 \beta \omega}\right) \psi
$$

and the solution for constant $\alpha$ and $\beta$ is of the form

$$
\psi=\exp \left( \pm \gamma \frac{\beta E^{1 / 2} \alpha}{k \omega^{2}+2 \beta \omega} r\right) \exp ( \pm i \gamma r)
$$

This implies a rapid oscillation of typical length scale $\gamma$

$$
\gamma \sim k \sqrt{|2 \beta|}
$$

modulated by a slow exponential decay with typical length scale $\delta$ given by

$$
\delta \sim \gamma^{-1} E^{-1 / 2} \alpha^{-1} \omega
$$

Inside this envelope, the radial length scale $\gamma^{-1}$ of the perturbation decreases when $\beta$ increases. It corresponds to the spiraling of the Rossby wave observed for variable $\beta$ (see fig. 3bc)

Using the scalings for the Stewartson layer instability $k \sim E^{-1 / 4}$ and $\omega \sim E^{1 / 4}$, relation B 8 leads to

$$
\delta \sim(2|\beta|)^{-1 / 2} \alpha^{-1} E^{0}
$$

which implies that the radial extension of the perturbation is independent of $E$. This is a fundamental difference with the thermal convection case investigated by Yano (1992) and Jones et al. (2000) where the scaling $k \sim E^{-1 / 3}$ and $\omega \sim E^{1 / 3}$ implies

$$
\delta \sim(2|\beta|)^{-1 / 2} \alpha^{-1} E^{1 / 6}
$$

which corresponds to a slowly decaying radial extension when decreasing $E$.

\section{B.3. Inner part, small $\beta$}

The inner part behaves exactly as the outer one, replacing $\omega$ by $\omega-R o U k$. For a spherical shell it corresponds to the small $\beta$ case.

If $|2 \beta|<k \omega$, we can rewrite $\mathrm{B} 5$ as

$$
\frac{\partial^{2} \psi}{\partial r^{2}}=\gamma^{2}\left(1-\frac{2 i \beta E^{1 / 2} \alpha}{k \omega^{2}+2 \beta \omega}\right) \psi
$$


which actually exchanges the length scale of the exponential decay and of the oscillation compared to the outer region. The solution for constant $\alpha$ and $\beta$ is then

$$
\psi=\exp ( \pm \gamma r) \exp \left(\mp i \gamma \frac{\beta E^{1 / 2} \alpha}{k \omega^{2}+2 \beta \omega} r\right)
$$

which is an exponential decay of characteristic length $\gamma^{-1}$, inside which there are much larger length scale oscillations, which cannot be detected.

For the Rossby wave, we have $\omega \sim k^{-1}$, so that $\gamma \sim k$, with a correction independent of $E$. This shows that in the small $\beta$ case the critical mode has a radial extension comparable to its azimuthal scale.

\section{Appendix C. Stability threshold data}

C.1. Split-sphere stability threshold

\begin{tabular}{ccccc}
$E$ & $R o_{c}$ & $m_{c}$ & $\omega_{c}$ & $\mathrm{NR}$ \\
\hline $10^{-5}$ & $1.40610^{-2}$ & 5 & $9.88610^{-2}$ & 300 \\
& $-1.79310^{-2}$ & 4 & $-3.49510^{-2}$ & \\
$10^{-6}$ & $3.32710^{-3}$ & 10 & $5.42810^{-2}$ & 400 \\
& $-3.87810^{-3}$ & 8 & $-2.06010^{-2}$ & \\
$10^{-7}$ & $8.14110^{-4}$ & 18 & $2.68910^{-2}$ & 600 \\
& $-9.35910^{-4}$ & 15 & $-0.99910^{-2}$ & \\
$10^{-8}$ & $2.21010^{-4}$ & 33 & $1.38810^{-3}$ & 800 \\
& $-2.45610^{-4}$ & 31 & $-6.44610^{-3}$ & \\
$10^{-9}$ & $6.37410^{-5}$ & 63 & $7.50510^{-3}$ & 1200 \\
& $-6.78310^{-5}$ & 61 & $-3.84410^{-3}$ & \\
$10^{-10}$ & $1.87510^{-5}$ & 121 & $4.12810^{-3}$ & 1500 \\
& $-1.94010^{-5}$ & 119 & $-2.31710^{-3}$ &
\end{tabular}

Linear stability threshold values for different Ekman numbers E. NR denotes the number of radial grid points used for the calculation. These values where obtained by QG numerical calculations with no-slip boundary conditions and including Ekman friction.

\section{C.2. Boundary conditions and viscosity dependence}

$$
\text { E ns,ep ns,ep0 fs,ep0 ns,ep,dv0 ns,ep,vs0 ns,ep, } \times 2
$$

\begin{tabular}{lllllll}
\hline $10^{-6}$ & 1.0 & 0.86 & 0.87 & 0.995 & 0.64 & 0.999 \\
$10^{-7}$ & 1.0 & 0.91 & 0.91 & 1.001 & 0.72 & 1.000 \\
$10^{-8}$ & 1.0 & 0.94 & 0.94 & 1.002 & 0.78 & 1.000
\end{tabular}

Comparison between $R o_{c}$ of different numerical models for the split-sphere geometry. Values are the $R o_{c}$ obtained by a model divided by the reference $R o_{c}(n s, e p)$ for $R o>0$ at a given Ekman number. The models are referenced by $n s$ and $f s$ respectively for noslip and free-slip boundary conditions, ep for the Ekman pumping, $d v$ for horizontal divergence and $v s$ for bulk viscosity; $\times 2$ stands for calculation with doubled number of radial grid points. A 0 denotes the absence of this feature. 


\section{C.3. Disks experimental threshold}

\begin{tabular}{ccc}
$E$ & stable $R o$ & unstable $R o$ \\
\hline $2.2310^{-5}$ & $3.5410^{-2}$ & $3.8910^{-2}$ \\
$1.1910^{-5}$ & $2.6410^{-2}$ & $2.8210^{-2}$ \\
$7.3010^{-6}$ & $1.9610^{-2}$ & $2.0810^{-2}$ \\
$4.8810^{-6}$ & $1.7010^{-2}$ & $1.7810^{-2}$ \\
$3.6810^{-6}$ & $1.4610^{-2}$ & $1.5210^{-2}$ \\
$3.0410^{-6}$ & $1.3010^{-2}$ & $1.4010^{-2}$ \\
\hline $2.3810^{-5}$ & $-4.3810^{-2}$ & $-5.2510^{-2}$ \\
$1.2310^{-5}$ & $-3.2410^{-2}$ & $-3.3410^{-2}$ \\
$9.2110^{-6}$ & $-2.4310^{-2}$ & $-2.5010^{-2}$ \\
$6.7010^{-6}$ & $-1.7210^{-2}$ & $-1.8210^{-2}$ \\
$4.9110^{-6}$ & $-1.6210^{-2}$ & $-1.7310^{-2}$ \\
$3.6810^{-6}$ & $-1.3810^{-2}$ & $-1.4010^{-2}$
\end{tabular}

Stability threshold experimental data obtained for the disks geometry, in water $(\nu=$ $10^{-6} \mathrm{~m}^{2} / \mathrm{s}$ ). See $\S 6.2$ for details about the experimental setup.

C.4. Spherical shell experimental threshold
$E$
\begin{tabular}{ccc} 
stable $R o$ & unstable $R o$ \\
\hline $3.5110^{-5}$ & $6.1310^{-2}$ & $6.4110^{-2}$ \\
$2.3010^{-5}$ & $4.7310^{-2}$ & $4.9410^{-2}$ \\
$1.2110^{-5}$ & $3.25210^{-2}$ & $3.34610^{-2}$ \\
$7.3010^{-6}$ & $2.42610^{-2}$ & $2.48510^{-2}$ \\
$4.8810^{-6}$ & $1.85510^{-2}$ & $1.93210^{-2}$ \\
$3.6710^{-6}$ & $1.56810^{-2}$ & $1.59610^{-2}$ \\
\hline $2.3010^{-5}$ & $-10.5710^{-2}$ & $-10.9610^{-2}$ \\
$1.2110^{-5}$ & $-8.1210^{-2}$ & $-8.6110^{-2}$ \\
$6.0910^{-6}$ & $-5.7810^{-2}$ & $-6.2710^{-2}$
\end{tabular}

Stability threshold experimental data obtained for the spherical shell geometry, in water $\left(\nu=10^{-6} \mathrm{~m}^{2} / \mathrm{s}\right)$. See $\S 6.2$ for details about the experimental setup.

\section{REFERENCES}

Aubert, J., Gillet, N. \& Cardin, P. 2003 Quasigeostrophic models of convection in rotating spherical shells. Geochem. Geophys. Geosyst. 4(7), 1052 doi:10.1029/2002GC000456.

Busse, F. H. 1968 Shear flow instabilities in rotating systems. J. Fluid Mech. 33, 577-589.

Busse, F. H. 1970 Thermal instabilities in rapidly rotating systems. J. Fluid Mech. 44, 441-460.

Cardin, P., Brito, D., Jault, D., Nataf, H.-C. \& Masson, J.-P. 2002 Towards a rapidly rotating liquid sodium dynamo experiment. Magnetohydrodynamics 38, 177-189.

CARdin, P. \& Olson, P. 1994 Chaotic thermal convection in a rapidly rotating spherical shell: consequences for flow in the outer core. Phys. Earth Planet. Inter. 82, 235.

Dormy, E., CARdin, P. \& JAUlt, D. 1998 MHD flow in a slightly differentially rotating spherical shell, with conducting inner core, in a dipolar magnetic field. Earth. Planet. Sc. Lett. 160, 15-30.

Dormy, E., Soward, A., Jones, C., Jault, D. \& Cardin, P. 2004 The onset of thermal convection in rotating spherical shells. J. Fluid Mech. 501, 43-70. 
FrüH, W.-G. \& READ, P. L. 1999 Experiments on a barotropic rotating shear layer. part 1. instability and steady vortices. J. Fluid Mech. 383, 143-173.

Greenspan, H. P. 1968 The theory of rotating fluids. Cambridge University Press.

Hide, R. \& Titman, C. W. 1967 Detached shear layers in a rotating fluid. J. Fluid Mech. 29, 39-60.

Hollerbach, R. 1994 Magnetohydrodynamic ekman and stewartson layers in a rotating spherical shell. Proc. R. Soc. London A 444, 333-346.

Hollerbach, R. 2003 Instabilities of the Stewartson layer part 1. The dependence on the sign of Ro. J. Fluid Mech. 492, 289-302.

Hollerbach, R., Futterer, B., More, T. \& Egbers, C. 2004 Instabilities of the Stewartson layer part 2. supercritical mode transitions. Theor. Comput. Fluid Dyn. .

Jones, C. A., SowArd, A. M. \& Mussa, A. I. 2000 The onset of thermal convection in a rapidly rotating sphere. J. Fluid Mech. 405, 157-179.

KIsS, A. E. 2003 A modified quasigeostrophic formulation for weakly nonlinear barotropic flow with large-amplitude depth variations. Ocean Model. 5, 171-191.

van de Konijnenberg, J. A., Nielsen, A. H., Rasmussen, J. J. \& Stenum, B. 1999 Shearflow instability in a rotating fluid. J. Fluid Mech. 387, 177-204.

Kuo, H. L. 1949 Dynamic instability of two-dimensional non-divergent flow in a barotropic atmosphere. J. Meteor. 6, 105-122.

Moore, D. W. \& Saffman, P. G. 1969 The structure of free vertical shear layers in a rotating fluid and the motion produced by a slowly rising body. Phil. T. R. Soc. Lond. A 264, 597-634.

NiIno, H. \& MisaWA, N. 1984 An experimental and theoretical study of barotropic instability. J. Atmos. Sci. 41, 1992-2011.

Noir, J., Cardin, P., Jault, D. \& Masson, J.-P. 2003 Experimental evidence of nonlinear resonance effects between retrograde precession and the tilt-over mode within a spheroid. Geophys. J. Int. 154, 407-416.

Pedlosky, J. 1987 Geophysical fluid dynamics, 2nd edn. Springer, New York.

Proudman, I. 1956 The almost-rigid rotation of viscous fluid between concentric spheres. $J$. Fluid Mech. 1, 505-516.

Roberts, P. H. \& Stewartson, K. 1963 On the stability of a Maclaurin spheroid of small viscosity. Astrophys. J. 137, 777-790.

SchaEfFER, N. 2004 Instabilits, turbulence et dynamo dans une couche de fluide cisaille en rotation. $\mathrm{PhD}$ thesis, Université Joseph Fourier.

Stewartson, K. 1957 On almost rigid rotations. J. Fluid Mech. 3, 17-26.

Stewartson, K. 1966 On almost rigid rotations. part 2. J. Fluid Mech. 26, 131-144.

YANO, J.-I. 1992 Asymptotic theory of thermal convection in rapidly rotating systems. J. Fluid Mech. 243, 103-131.

YAno, J.-I., TAlagrand, O. \& Drossart, P. 2003 Outer planets: Origins of atmospheric zonal winds. Nature 421, 36.

Zavala Sanson, L. \& van Heisst, G. J. F. 2002 Ekman effects in a rotating flow over bottom topography. J. Fluid Mech. 471, 239-255.

ZhANG, K. K. 1992 Spiralling columnar convection in rapidly rotating spherical fluid shells. J. Fluid Mech. 236, 535-556. 